\title{
Transient and Chronic Neonatal Denervation of Murine Muscle: A Procedure to Modify the Phenotypic Expression of Muscular Dystrophy
}

\author{
Maria C. Moschella and Marcia Ontell \\ Department of Neurobiology, Anatomy and Cell Science, University of Pittsburgh, School of Medicine, Pittsburgh, \\ Pennsylvania 15261
}

\begin{abstract}
The extensor digitorum longus muscles of 14-d-old normal $(129 \operatorname{ReJ}++)$ and dystrophic (129 ReJ dy/dy) mice were denervated by cutting the sciatic nerve. One denervation protocol was designed to inhibit reinnervation of the shank muscles, the other to promote reinnervation. Chronically denervated muscles (muscles that remained denervated for $100 \mathrm{~d}$ after nerve section) exhibited marked atrophy, but the number of myofibers in these muscles (1066 \pm 46 and $931 \pm$ 62 for the denervated normal and dystrophic muscles, respectively) was similar to the number of myofibers found in age-matched, unoperated normal muscles [ $922 \pm 28$ (Ontell et al., 1984)] and was significantly greater than the number of myofibers found in age-matched dystrophic muscles [547 \pm 45 (Ontell et al., 1984)]. Similar effects on myofiber number were obtained when denervated muscles were allowed to reinnervate. Reinnervation of both normal and dystrophic muscles mitigated the marked atrophy that characterized chronically denervated muscles. The dystrophic reinnervated muscles appeared "healthier" than agematched, unoperated dystrophic muscles, having $70 \%$ more myofibers, less myofiber diameter variability, substantially less connective tissue infiltration, and a greater amount of contractile tissue at their widest girths. The present study demonstrated that it is possible to alter the phenotypic expression of the histopathological changes associated with murine dystrophy, in dystrophic myofibers that are formed during fetal development, by subjecting the muscle to neonatal denervation.
\end{abstract}

Murine muscular dystrophy (dy) is a progressive, hereditary disorder (Michelson et al., 1955), clinical symptoms (i.e., hindlimb dragging) being observed when the mice are approximately 2 weeks of age. The disorder is characterized by myofiber necrosis and an abortive attempt at muscle regeneration, ultimately resulting in extensive myofiber loss and replacement by connective tissue.

Despite extensive biochemical, morphological, and physio-

Received July 25, 1986; revised Jan. 12, 1987; accepted Jan. 23, 1987

This work was supported by NIH Grant AR36294, a grant from the Muscular Dystrophy Association, and the Health Research and Services Foundation. For assistance in statistical evaluation, we wish to thank Dr. Floyd Taylor, Department of Community Medicine, University of Pittshurgh School of Medicine. The competent technical assistance of Ms. G. Diluiso is acknowledged.

Correspondence should be addressed to Marcia Ontell, Ph.D., Department of Neurobiology, Anatomy and Cell Science, University of Pittsburgh, School of Medicine, 3550 Terrace Street, Pittsburgh, PA 15261.

Copyright (C) 1987 Society for Neuroscience $0270-6474 / 87 / 072145-08 \$ 02.00 / 0$ logical studies, the etiology of the disease has not been determined. Initially, the disease was described as a primary myopathy (Michelson et al., 1955; Banker and Denny-Brown, 1959; Banker, 1967; Cosmos et al., 1973); however, amyelinization of the ventral roots of the spinal nerves in the lumbar region (Bray and Aguayo, 1975; Jaros and Bradley, 1978), abnormalities at the motor endplate region (Banker et al., 1979), and other neurological changes were subsequently described.

Recently our laboratory has been able to modify the phenotypic expression of murine muscular dystrophy in regenerated myofibers that form after the removal, and replacement, of whole young dystrophic muscle back into its original bed (Bourke and Ontell, 1986). Subsequent to transplantation, the myofibers of the ischemic grafted muscle undergo necrosis, phagocytosis, and replacement by newly formed multinucleated myotubes (Carlson, 1973; Carlson and Gutmann, 1976). The number of myofibers found in the dystrophic grafts $100 \mathrm{~d}$ after transplantation is the same as the number found in similarly treated normal muscles. The wide myofiber diameter variation and extensive connective tissue infiltration that are characteristic of untraumatized dystrophic muscle are greatly reduced in dystrophic grafts, and the grafts appear "healthier" than age-matched, unoperated dystrophic muscles (Bourke and Ontell, 1986).

The ability to modify the phenotypic expression of dystrophy in regenerated dystrophic muscles maintained in a dystrophic environment and innervated by dystrophic nerves appears to contradict both the myogenic and neurogenic hypotheses concerning the etiology of the disorder. If the myogenic hypothesis is correct, one might expect the regenerated dystrophic myofibers, being formed by "dystrophic" myosatellite cells (myogenic stem cells; Mauro, 1961), to undergo the same histopathological changes as occur in dystrophic myofibers formed during fetal development. If the neurogenic hypothesis is correct, one might expect the fate of the regenerated dystrophic myofibers, being innervated by "dystrophic" nerves, to be similar to that observed in untraumatized dystrophic muscle.

In an effort to understand the mechanism responsible for the modification of the dystrophic syndrome subsequent to transplantation, differences between primary myogenesis (fetal myogenesis) and secondary myogenesis (myogenesis subsequent to transplantation) have been examined. One difference involves the time of myoneural integration, relative to the time of myotube formation. During primary myogenesis of normal mouse muscle, myotubes become innervated within a day or two of their formation (Ontell and Kozeka, 1984). By contrast, there is a delay of at least a week between myotube formation and 
the appearance of the first myoneural junctions in the transplantation system (Bourke and Ontell, 1986). Perhaps the delay in innervation of the regenerated myofibers found in the dystrophic graft could be responsible for the modification of the dystrophic myofibers' phenotype. It is suggested that genetically dystrophic myofibers will exhibit histopathological changes consistent with murine dystrophy only if they are, and remain, innervated during a "critical" period following myotube formation. To test this hypothesis, our laboratory has undertaken an experiment in which dystrophic muscle has been induced to regenerate while the time course of its reinnervation was altered, and an experiment in which the innervation of fetally formed dystrophic muscle has been interrupted during the neonatal period. This report concerns the results of the study in which innervation of dystrophic muscle has been permanently or transiently interrupted during the neonatal period.

\section{Materials and Methods}

The right extensor digitorum longus muscles of 2-week-old female 129 $\mathrm{ReJ}$ dy/dy mice and of 2-week-old female $129 \mathrm{ReJ}$ (genetically normal) mice (derived from colonies maintained at the Jackson Laboratory) werc denervated by cutting and removing $1.5 \mathrm{~mm}$ of the sciatic nerve as it passed under the biceps femoris muscle. The proximal end of the cut nerve stump was folded back on itself in an attempt to prevent reinnervation of the shank musculature. One hundred days post-denervation, the region under the biceps femoris muscle was examined to evaluate whether any of the sprouting nerves from the proximal nerve stump were growing in the direction of the denervated shank. The right extensor digitorum longus muscles of a second group of 2-week-old female 129 $\mathrm{ReJ} \mathrm{dy/dy} \mathrm{mice} \mathrm{and} \mathrm{2-week-old} \mathrm{female} 129 \mathrm{ReJ}$ (genetically normal) mice were denervated by carefully severing the sciatic nerve, with minimal disturbance to the surrounding fascia, as the nerve passed under the biceps femoris muscle. The cut ends of the nerves were closely approximated to facilitate reinnervation of the shank musculature. One hundred days after denervation, the sciatic nerve was cxposed in order to determine whether the gap between the cut edges of the nerves had been eliminated.

One hundred days after denervation, denervated muscles and contralateral, unoperated extensor digitorum longus muscles were exposed and fixed in situ in $2.0 \%$ glutaraldehyde in $0.125 \mathrm{M}$ cacodylate buffer (pH 7.3) for $30 \mathrm{~min}$. Extensor digitorum longus muscles of 2 -week-old and 17-week-old untraumatized normal and dystrophic animals were similarly treated. All muscles were removed from the hindlimb and placed in fresh fixative for $1.5 \mathrm{hr}$. Muscles were postfixed in buffered $2.0 \%$ osmium tetroxide, dehydrated in ethanol, and embedded in Epon 812. Epon blocks of muscles were placed on a sliding microtome (equipped with a steel knife) and oriented for sectioning in a plane perpendicular to the long axis of the muscle fibers. (The extensor digitorum longus muscle is a pennate muscle, and a planc perpendicular to the long axis of the myofibers is not perpendicular to the long axis of the muscle.) The muscles were serially sectioned, from origin to insertion, into sets consisting of ten $15-\mu \mathrm{m}$-thick sections and one 8- $\mu \mathrm{m}$ thick section. Fifteen-micron-thick sections were cleared in drops of Epon between 2 layers of polystyrene film and placed in a $60^{\circ} \mathrm{C}$ oven (Davidowitz et al., 1976). The 8- $\mu$ m-thick sections were mounted on glass slides. All 8- $\mu \mathrm{m}$-thick sections and all cleared sections of the chronically denervated dystrophic muscles were examined, using a Leitz microscope equipped with phase optics, to determine whether they contained myelinated nerves. The maximal cross-sectional areas of all muscles (hereafter termed the muscles' "widest girths") were determined by examination of each $8-\mu$ m-thick section with a phase microscope equipped with an eyepiece reticule. The $15-\mu \mathrm{m}$-thick section adjacent to the muscle's widest girth was cut out of the polystyrene film and attached to a preformed Epon block. Other randomly chosen $15-\mu \mathrm{m}$ thick sections were also reembedded. Reembedded sections were cut into semithin $(0.5 \mu \mathrm{m})$ or ultrathin $(70-90 \mathrm{~nm})$ sections. Semithin sections were stained with toluidine blue. Ultrathin sections were collected on slot grids, stained with uranyl acetate and leaf citrate (Reynolds, 1963), and observed using a Philips 300 electron microscope. Muscles believed to be chronically denervated were examined, using the electron microscope, to determine whether axons could be identified in ultrathin sections. Muscles believed to be denervated-reinnervated were examined to establish whether there were motor endplates on the myofibers.

Montages of micrographs $(\times 1100$; microscope and enlarger were calibrated at each use) of the entire cross-section of the muscle, taken at the muscle's widest girth, were made. Morphometric analyses were performed on electron micrographs of chronically denervated $(N=3$ for denervated normal muscle, $N=3$ for denervated dystrophic muscle) and on denervated-reinnervated $(N=5$ for denervated-reinnervated normal muscle, $N=5$ for denervated-reinnervated dystrophic muscle) muscles $100 \mathrm{~d}$ post-denervation, and on age-matched normal and dystrophic muscles ( $N=3$ for normal, and $N-4$ for dystrophic muscles). The number of myofibers present at the widest girth of each muscle was determined from the montages of electron micrographs. With a Bioquant-IBM morphometric analysis system, the perimeters of 300 randomly chosen myofibers per muscle were traced with a digitizing pen. A calculation program determined the diameter of each myofiber (assuming each myofiber to be circular in cross-section). The mean myofiber diameters and histograms of myofiber diameter distributions were calculated by the Bioquant system for each muscle and for all muscles in a given group. The total amount of contractile tissue at the muscle's widest girth was determined by multiplying the number of myofibers in each muscle by the mean myofiber area for that muscle. Morphometric data generated from chronically and transiently denervated normal and dystrophic muscles were compared to data from age-matched, untraumatized normal and dystrophic muscles generated in this study and in Ontell et al. (1984). Data were subjected to statistical analysis using a one-way analysis of variance, followed by paired comparisons using Student's $t$ test (Sokal and Rohlf, 1981). An analysis of variance of myofiber number was made using the square root of the number of myofibers. To determine whether the changes in variance in fiber diameter in denervated-reinnervated muscle, compared to those in unoperated muscle, were significant, the variances observed in the 2 groups were compared using an $F$ ratio (Sokal and Rohlf, 1981). All references to significant differences in this report indicate significance at $p<0.01$, unless other levels of significance are specified. The ratio of centrally and eccentrically located myonuclei to subsarcolemmal myonuclei in chronically and transiently denervated muscles was determined by observation of the location of myonuclei, in 250 myofibers per muscle, in ultrathin scctions taken through the muscles' widest girths. Central nuclei may serve as markers for the determination of the extent of regeneration (i.e., the number of regenerating myofibers) in murine muscle (see Discussion).

\section{Results}

\section{Unoperated dystrophic muscle}

The effect of dystrophy on the extensor digitorum longus muscle of the $129 \mathrm{ReJ}$ dy/dy mouse has been previously described (Ontell et al., 1984); therefore, only a brief summary of those histopathological changes necessary to evaluate the effect of denervation on dystrophic muscle will be given.

Al 14-d postnatal, dystrophic mice began to exhibit intermittent hindlimb dragging. At this stage the extensor digitorum longus muscle contained approximately $77 \%$ of the myofibers found in age-matched normal muscle (Ontell et al., 1984). Three types of myofibers could be identified on the basis of their selective response to the dystrophic process: polygonally shaped "healthy" myofibers, similar in appearance to myofibers found in normal muscle; swollen necrotic myofibers; and small-diameter myofibers, which, by ultrastructural criteria (central nuclei, varying amounts of myofibrils, glycogen deposits, etc.), were clearly regenerating myofibers (Fig. 1). This was the appearance of the muscle at the time of denervation.

At 17 weeks, the hindlimbs of the dystrophic mice were dragged passively behind their bodies, and the extensor digitorum longus muscle exhibited marked histopathological changes (Fig. 2). There was greater myofiber diameter variation, less well-defined fascicular arrangement, and more connective tissue infiltration than was found in age-matched normal muscle (Figs. 2 and 3), 

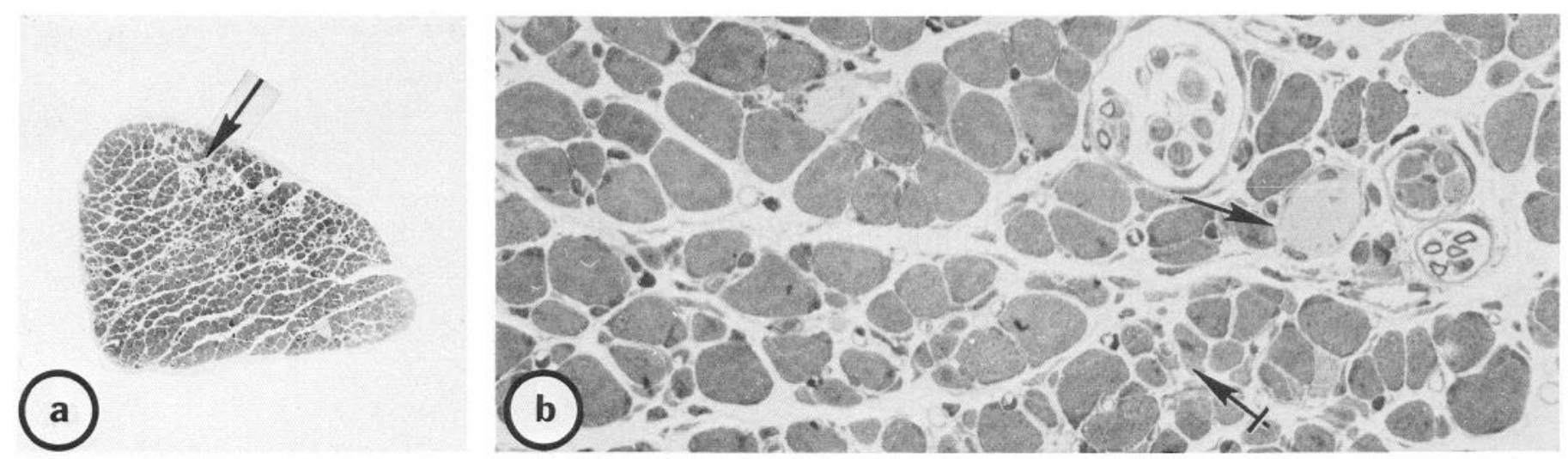

Figure 1. Light micrographs of the extensor digitorum longus muscle of a 2-week-old dystrophic mouse. Area indicated by arrow (a) is enlarged in $b$. There are occasional necrotic myofibers (arrow, $b$ ) and some small-diameter regenerating myofibers with central nuclei (crossed arrow, $b$ ). $a$ and $b$, Both are stained with Toluidine blue. $a, \times 60 ; b, \times 500$.

and the muscle contained approximately $41 \%$ fewer myofibers (Ontell et al., 1984).

\section{Chronically denervated muscle}

Gross observation of the region under the biceps femoris muscle, light-microscopic examination of all serial sections of each muscle, and randomly chosen ultrathin sections taken along the lengths of the muscles confirmed that the 3 normal and dystrophic, chronically denervated muscles contained no axons. Long-term, chronically denervated normal muscles (not shown) and dystrophic muscles (Fig. 4) were significantly $(p<0.02)$ smaller in cross-sectional area than were the respective unoperated muscles, with denervated dystrophic muscles being significantly smaller in cross-sectional area than denervated normal muscles (Table 1). All chronically denervated muscles showed some evidence of regional connective tissue infiltration. Interfascicular connective tissue infiltration appeared to be more prominent in the chronically denervated dystrophic muscle; however, no quantitative evaluation was made. The number of myofibers found at the widest girths of the denervated normal and dystrophic muscles was similar in both, and also to the number of myofibers found in age-matched, unoperated normal muscles (Table 1). Chronically denervated dystrophic muscles contained a similar number of myofibers as was present in the dystrophic muscle at the time of denervation (Table 1), which suggests that denervation had resulted in the failure of the muscle to undergo the loss of myofibers characteristic of dystrophy. The atrophy of the chronically denervated muscles, as compared to control muscles, was the result of significant decreases in myofiber diameters (Fig. 5, Table 1). Denervation resulted in significant decreases in the cross-sectional areas of contractile tissue (number of myofibers $\times$ mean myofiber cross-sectional area) found at the muscles' widest girths (Table 1).

Central or eccentrically located myonuclei accounted for 3.5 and $6.5 \%$ of the total nuclear population in chronically denervated normal and dystrophic muscles, respectively. This could be interpreted as indicating that little, if any, myofiber regeneration occurred in the chronically denervated muscles (see Discussion).

\section{Denervated-reinnervated muscle}

Both normal and dystrophic mice in the denervated-reinnervated groups failed to make full use of their reinnervated limbs, even after $100 \mathrm{~d}$ following denervation. Examination of the sciatic nerve in the region of the original nerve lesion established that the gap between the proximal and distal nerve stumps had
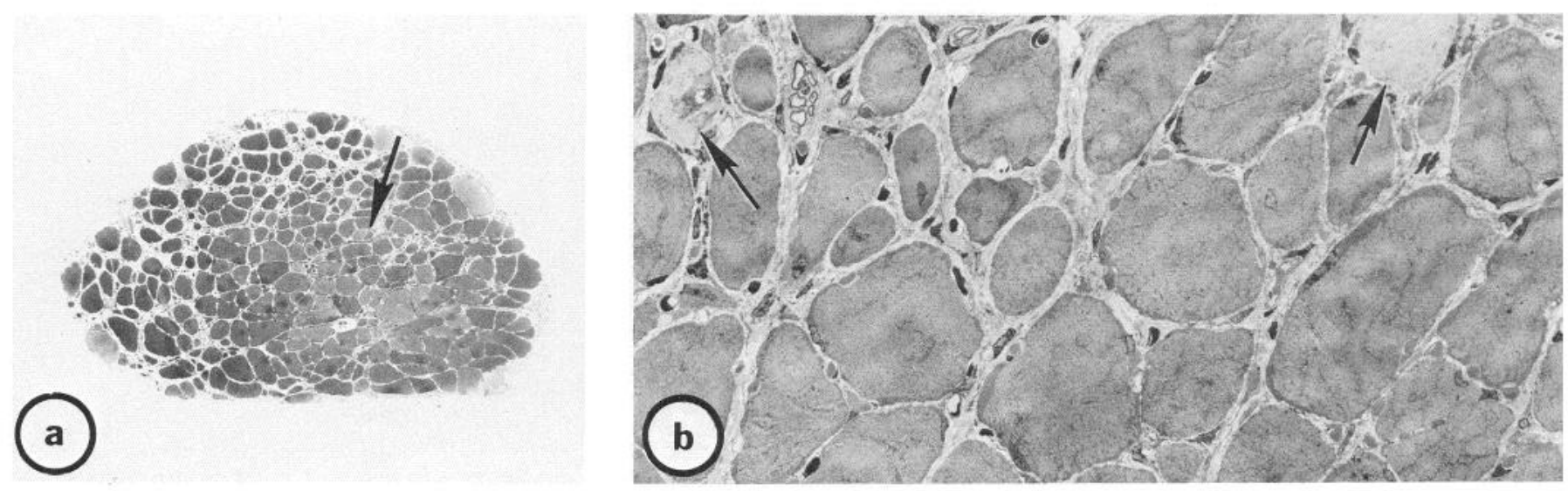

Figure 2. Extensor digitorum longus muscle of an unoperated 17-week-old dystrophic mouse. Area indicated by arrow on the light micrograph $(a)$ is enlarged in the electron micrograph $(b) . b$, Arrows indicate necrotic myofibers. At this stage there is marked myofiber diameter variation and extensive connective tissue infiltration. $a$, Toluidine blue. $\times 60 . b$, Uranyl acetate and lead citrate. $\times 600$. 

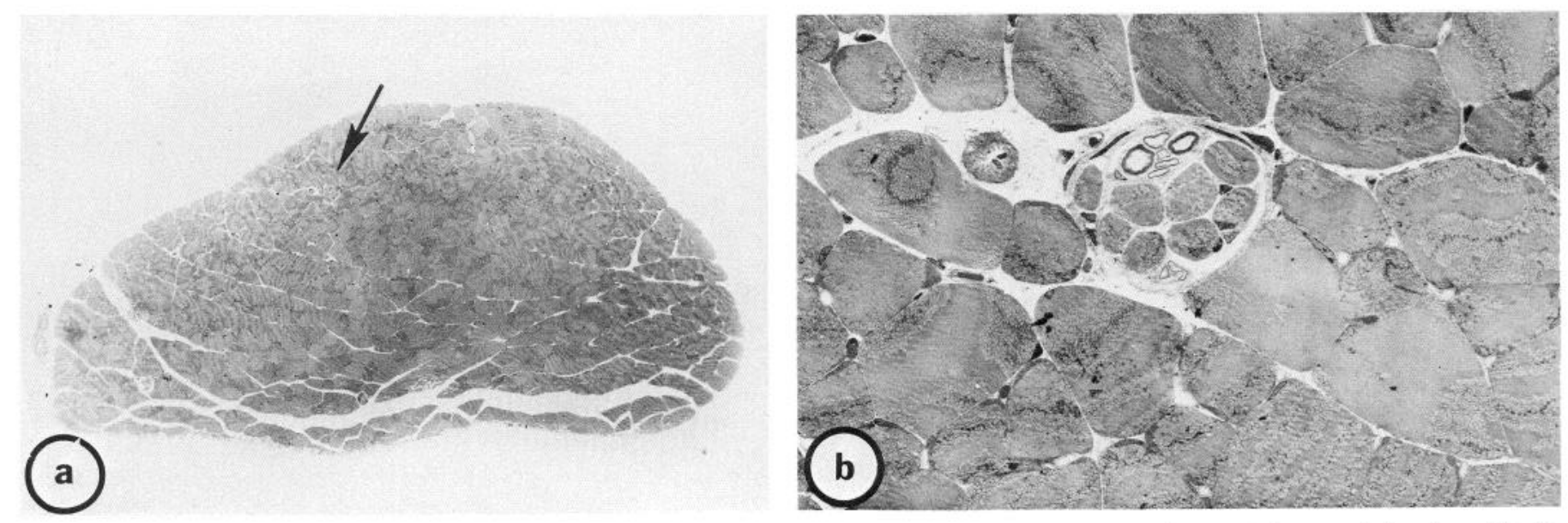

Figure 3. Normal, unoperated extensor digitorum longus muscle of a 17-week-old mouse. Area in light micrograph (a, arrow) is enlarged in the electron micrograph $(b)$. Compare the appearance of these myofibers with that of the myofibers found in normal (Fig. $6 b$ ) and dystrophic (Fig. $7 b$ ) denervated-reinnervated muscle. $a$, Toluidine blue. $\times 60$. $b$, Uranyl acetate and lead citrate. $\times 600$.

been eliminated. Myelinated nerve bundles (Fig. 6c) and motor endplates (Figs. 8, 9) were seen in each of the 5 normal and dystrophic muscles in each group. While no morphometric analyses of motor endplate morphology were undertaken, the dystrophic motor endplates (Fig. 8) consistently displayed less well-developed soleplate infoldings than were found in normal reinnervated muscles (Fig. 9).

The appearance of the normal and dystrophic denervatedreinnervated muscles (Figs. 6, 7) was similar, and it was also similar to that of unoperated normal muscles (Fig. 3), except for the fact that the myofibers of the dystrophic denervatedreinnervated muscles were smaller than those of similarly treated and unoperated normal muscles. (Compare Figs. $3 b, 6 b$, and $7 b$.) Both groups of denervated-reinnervated muscles contained a similar number of polygonally shaped myofibers, and the number of myofibers in these muscles was similar to the number found in age-matched, unoperated normal muscles (Table 1). The morphological appearance of denervated-reinnervated dystrophic muscles was different than that of age-matched, unoperated dystrophic muscle. (Compare Figs. 2 and 7.) Denervated- reinnervated dystrophic muscles did not have the extensive connective tissue infiltration that characterized untraumatized dystrophic muscle. These muscles were significantly larger in girth $(p<0.02)$, had significantly more myofibers $(70 \%)$ and contractile tissue $(37 \%)$ at their widest girths than did unoperated dystrophic muscles (Table 1). Comparison of the histograms of myofiber diameter distributions of denervated-reinnervated dystrophic muscles and unoperated dystrophic muscles indicated that the dystrophic muscles failed to display the marked myofiber diameter variation characteristic of unoperated dystrophic muscles $(p<0.001)$. Both the large-diameter (probably work-hypertrophied) myofibers and the small-diameter (regenerating) myofibers characteristic of dystrophy were not found in the denervated-reinnervated dystrophic muscles (Fig. 7).

Approximately $2.5 \%$ of the myonuclei found in denervatedreinnervated normal muscle and $6.7 \%$ of the myonuclei found in denervated-reinnervated dystrophic muscle were centrally or eccentrically located, indicating that little, if any, myofiber regeneration occurred in the denervated-reinnervated muscles (see Discussion).
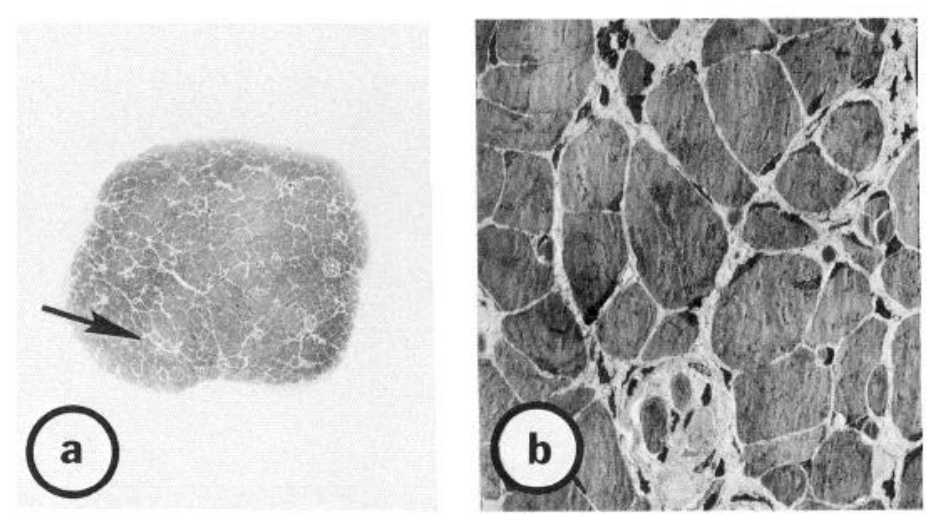

Figure 4. Chronically denervated extensor digitorum longus muscle of a dystrophic mouse. Denervation was performed when the mouse was 2 weeks old, and the muscle was removed $100 \mathrm{~d}$ later. The muscle has undergone atrophy $(a)$, as compared to the unoperated, age-matched dystrophic muscle (Fig. $2 a$ ). Area indicated by arrow $(a)$ is enlarged in the electron micrograph $(b)$. This atrophy is due to the small diameter of the myofibers in the chronically denervated muscles, rather than to a reduction in myofiber number. Compare the size of the myofibers in the electron micrograph $(b)$ with that in unoperated, age-matched dystrophic muscles (Fig. 2b) and in denervated-reinnervated dystrophic muscles (Fig. $7 b$ ). $a$, Toluidine blue. $\times 60 . b$, Uranyl acetate and lead citrate. $\times 600$. 


\section{Discussion}

The present study establishes, for the first time, that the phenotypic expression of genetically determined murine dystrophy in dystrophic myofibers formed during fetal development can be modified by neonatal denervation, followed by reinnervation. Denervated-reinnervated dystrophic muscles are of greater girth, contain $70 \%$ more myofibers and display $37 \%$ more contractile tissue at their widest girths than do untraumatized dystrophic muscles. These muscles show no evidence of necrotic changes and fail to display the marked myofiber diameter variation and connective tissue infiltration that occur in dystrophic muscles of age-matched mice. Moreover, denervated-reinnervated dystrophic muscles have similar numbers of myofibers and are similar in overall appearance to denervated-reinnervated normal muscles and age-matched, unoperated normal muscles. However, on all other parameters measured (maximal crosssectional area, mean myofiber diameter, and contractile tissue area at the muscles' widest girths), the reinnervated dystrophic muscles are smaller than the muscles in the normal groups. The smaller size of the dystrophic denervated-reinnervated muscles may reflect the reduced body weight of dystrophic mice compared to normal mice (Rowe and Goldspink, 1969a, b).

We have previously shown (Bourke and Ontell, 1986) that it is possible to modify the expression of murine dystrophy by transplanting young whole dystrophic muscles into a dystrophic host. The transplantation system differs from the neonatal denervation-reinnervation system in that the genetically dystrophic myofibers that fail to express the dystrophic phenotype in the transplantation system are regenerated myofibers (myofibers that were formed de novo subsequent to the extreme trauma of grafting), while the myofibers in the denervated-reinnervated dystrophic muscle were formed during fetal development. Moreover, the denervation-reinnervation protocol produces a "healthier"-appearing muscle, one that is larger in girth, contains $47 \%$ more myofibers, and has a cross-sectional area of contractile tissue that is $2 \frac{1}{2}$ times greater than that of dystrophic grafted muscle (Bourke and Ontell, 1986; D. L. Bourke, unpublished observations).

That the chronically denervated and the denervated-reinnervated dystrophic muscles contain $70 \%$ more myofibers than are found in unoperated dystrophic muscles suggests either that the
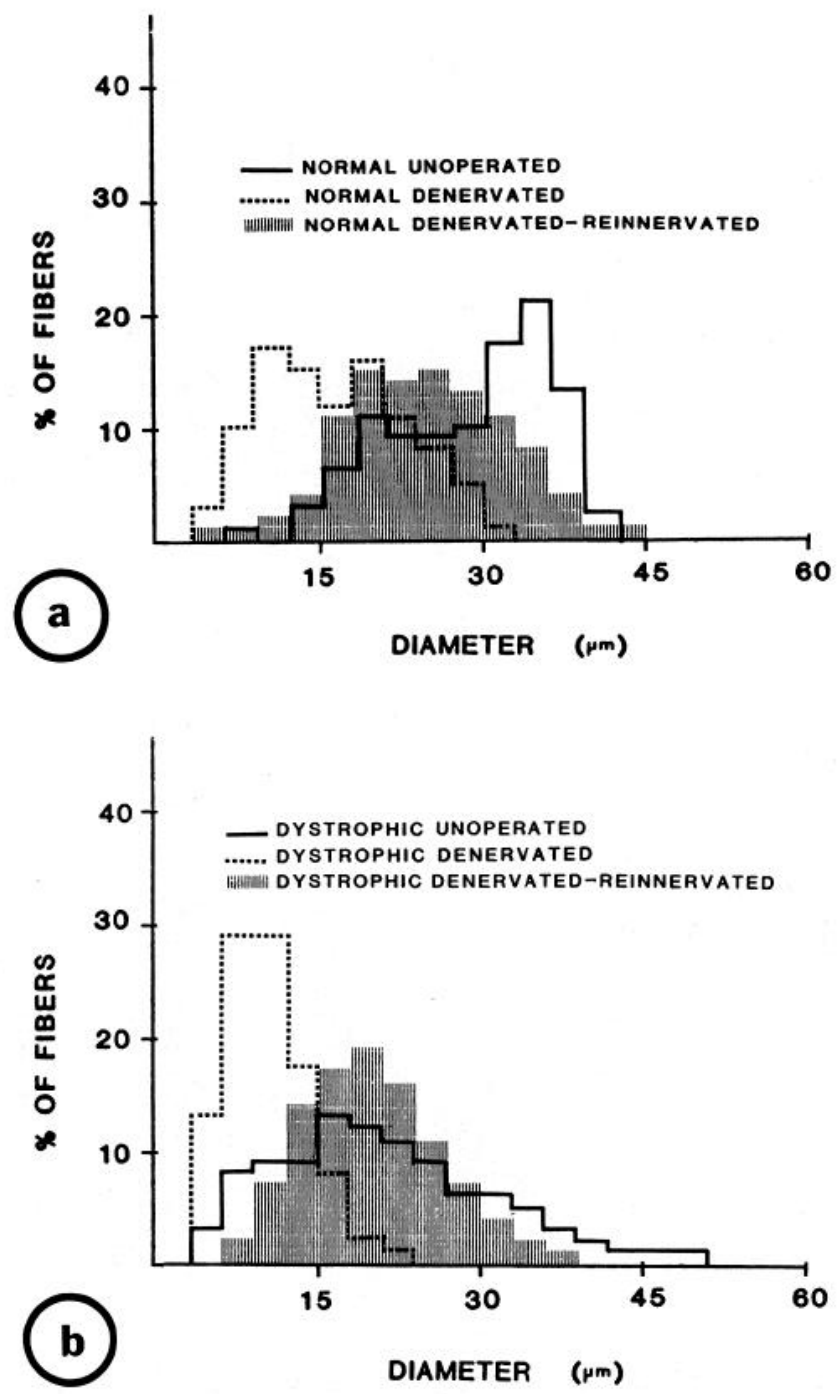

Figure 5. Histograms of myofiber diameter distributions, measured at the muscles' widest girths. $a$, Comparison of myofiber diameter distributions of unoperated normal, chronically denervated normal, and denervated-reinnervated normal muscles. $b$, Comparison of myofiber diameter distributions of unoperated dystrophic, chronically denervated dystrophic, and denervated-reinnervated dystrophic muscles.

Table 1. Morphometric analysis of chronically denervated and denervated-reinnervated extensor digitorum longus (EDL) muscles and their agematched control muscles ${ }^{a}$

\begin{tabular}{|c|c|c|c|c|c|c|}
\hline \multirow[b]{2}{*}{ Area } & \multicolumn{3}{|l|}{ Normal muscle } & \multicolumn{3}{|c|}{ Dystrophic muscle } \\
\hline & $\begin{array}{l}\text { Control }^{a} \\
17 \text {-wk-old } \\
(n=3)\end{array}$ & $\begin{array}{l}\text { Chronically } \\
\text { denervated }{ }^{c} \\
(n=3)\end{array}$ & $\begin{array}{l}\text { Denervated- } \\
\text { reinnervated }{ }^{d} \\
(n=5)\end{array}$ & $\begin{array}{l}\text { Control }^{a} \\
17 \text {-wk-old } \\
(n=4)\end{array}$ & $\begin{array}{l}\text { Chronically } \\
\text { denervated } \\
(n=3)\end{array}$ & $\begin{array}{l}\text { Denervated- } \\
\text { reinnervated }{ }^{d} \\
(n=5)\end{array}$ \\
\hline $\begin{array}{l}\text { Maximal cross-sectional } \\
\text { area of EDL }\left(\mathrm{mm}^{2}\right)\end{array}$ & $0.72 \pm 0.07$ & $0.51 \pm 0.02$ & $0.74 \pm 0.08$ & $0.35 \pm 0.01$ & $0.15 \pm 0.01$ & $0.53 \pm 0.03$ \\
\hline Number of myofibers & $922 \pm 28^{b}$ & $1066 \pm 46$ & $932 \pm 26$ & $547 \pm 45^{b}$ & $931 \pm 62$ & $928 \pm 35$ \\
\hline Mean myofiber diameter $(\mu \mathrm{m})$ & $28.4 \pm 0.3$ & $16.4 \pm 0.86$ & $24.7 \pm 0.89$ & $21.0 \pm 0.6$ & $10.2 \pm 0.29$ & $20.2 \pm 1.12$ \\
\hline $\begin{array}{l}\text { Total contractile tissue area } \\
\text { at } \mathrm{MWG}^{e}\left(\mathrm{~mm}^{2}\right)\end{array}$ & $0.624 \pm 0.02$ & $0.265 \pm 0.03$ & $0.482 \pm 0.02$ & $0.230 \pm 0.01$ & $0.087 \pm 0.002$ & $0.314 \pm 0.014$ \\
\hline
\end{tabular}

aAll values measured at muscles' widest girths (MWG) and expressed as means \pm SEM.

${ }^{b}$ Values taken from Ontell et al. (1984).

c Denervated at 2 weeks; removed $100 \mathrm{~d}$ after denervation.

- Denervated at 2 weeks and allowed to reinnervate. Muscles removed $100 \mathrm{~d}$ after denervation.

- Total contractile area $=$ mean number of myofibers $\times$ mean cross-sectional area of myofibers. 

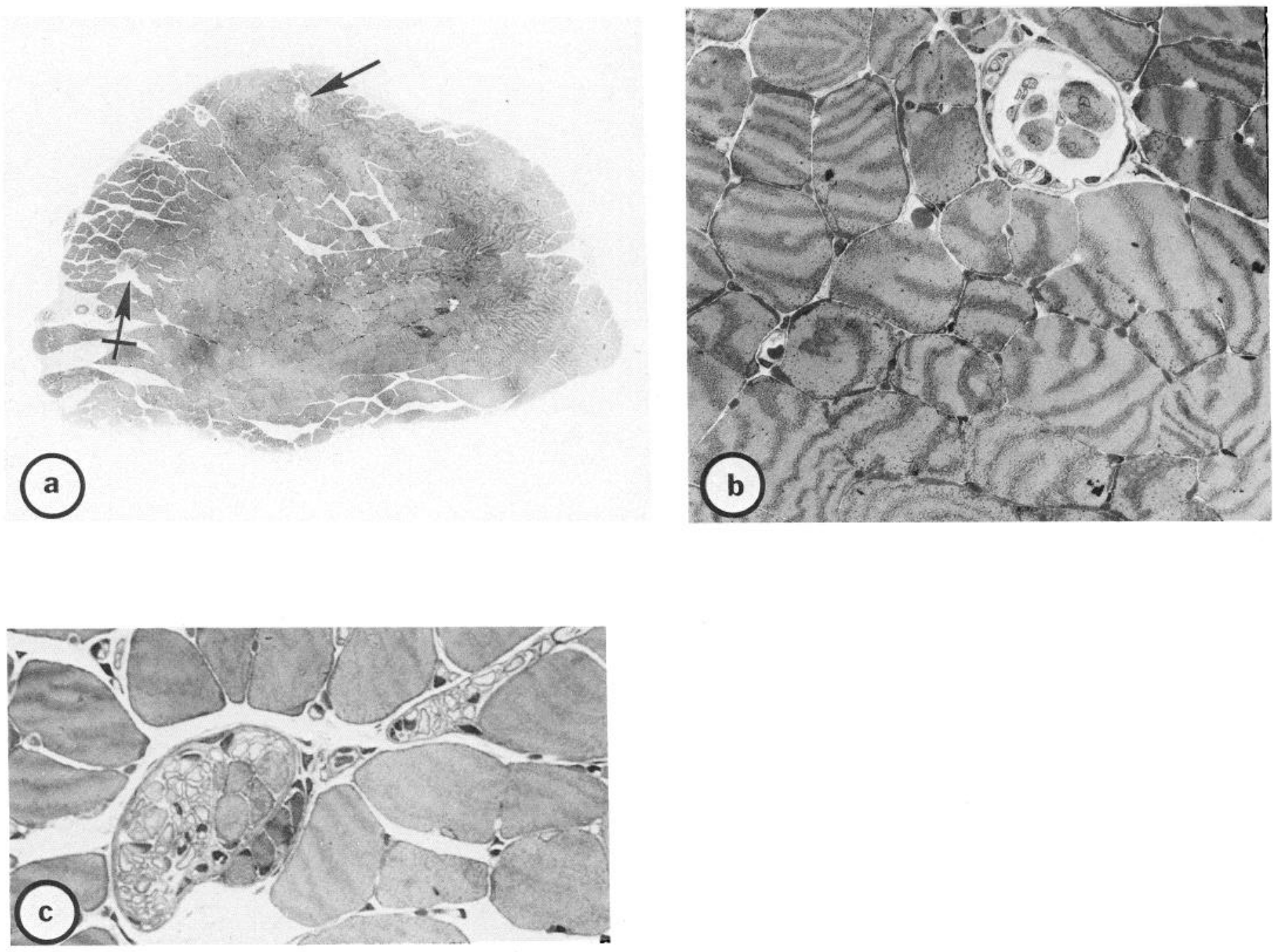

Figure 6. Denervated-reinnervated normal extensor digitorum longus muscle. Denervation was performed on a 2-week-old mouse, and the muscle was removed $100 \mathrm{~d}$ after denervation. Compare with age-matched, normal muscle (Fig. 3 ) and with denervated-reinnervated dystrophic muscle (Fig. 7). Area indicated by arrow in the light micrograph $(a)$ is enlarged in the electron micrograph $(b)$. Area indicated by crossed arrow (a) contains a myelinated nerve bundle $(c) . a$, Toluidine blue. $\times 45 . b$, Uranyl acetate and lead citrate. $\times 600 . c$, Toluidine blue. $\times 500$.

original myofibers have failed to undergo necrosis [the number of myofibers in the experimental muscles is similar to the number of fibers present in the dystrophic muscle at the time of denervation ( $965 \pm 37$ myofibers); Ontell et al., 1984] or that denervation has induced regeneration, resulting in the replacement of as many myofibers as might be lost from dystrophic muscle during this period. We favor the hypothesis that denervation has inhibited myofiber loss on the basis of our failure to find evidence of necrosis in chronically denervated dystrophic muscle at 2, 4, and 8 weeks after nerve section (M. C. Moschella, unpublished observations) and on the low frequency of central nuclei ( $<7 \%$ of the myonuclei) in all our experimental groups. In 2 systems characterized by extensive regeneration [wholemuscle transplantation (D. L. Bourke, unpublished observations) and regeneration following injection of bupivacaine $(\mathrm{H}$. Martin, unpublished observations)], $47-60 \%$ of the myonuclei in regenerated normal and dystrophic muscles remains centrally located $100 \mathrm{~d}$ after the onset of regeneration. If the frequency of central myonuclei serves as a clue to the extent of regeneration, regeneration does not play a significant role in maintaining myofiber number in chronically or transiently denervated muscles. Support for this hypothesis is found in Jaros and Johnstone (1984), who, having studied the effects of neonatal denervation on the dystrophic extensor digitorum longus muscle for periods of 1-2 weeks after nerve section, report an inhibition of myofiber loss and an absence of necrotic and regenerating myofibers, and in Karpati et al. (1982), who report that chronically denervated dystrophic hamster muscle does not display necrotic changes.

It is difficult to compare the effects of chronic and transient denervation on the expression of the dystrophic phenotype, other than to state that both systems are equally effective in preventing myofiber loss. Chronic denervation of muscle causes marked changes in muscle morphology, making it difficult to distinguish those changes in the muscle that are due to denervation from those that are due to dystrophy.

In the present study, the time course of reinnervation of the dystrophic muscle has not been determined. However, on the basis of the findings that the nerve is severed $9-10 \mathrm{~mm}$ from the point at which it enters the muscle, that the nerve stump grows at approximately $2.5 \mathrm{~mm} / \mathrm{d}$ after an initial refractory period (Gutmann, 1942), and that neonatally denervated muscle 

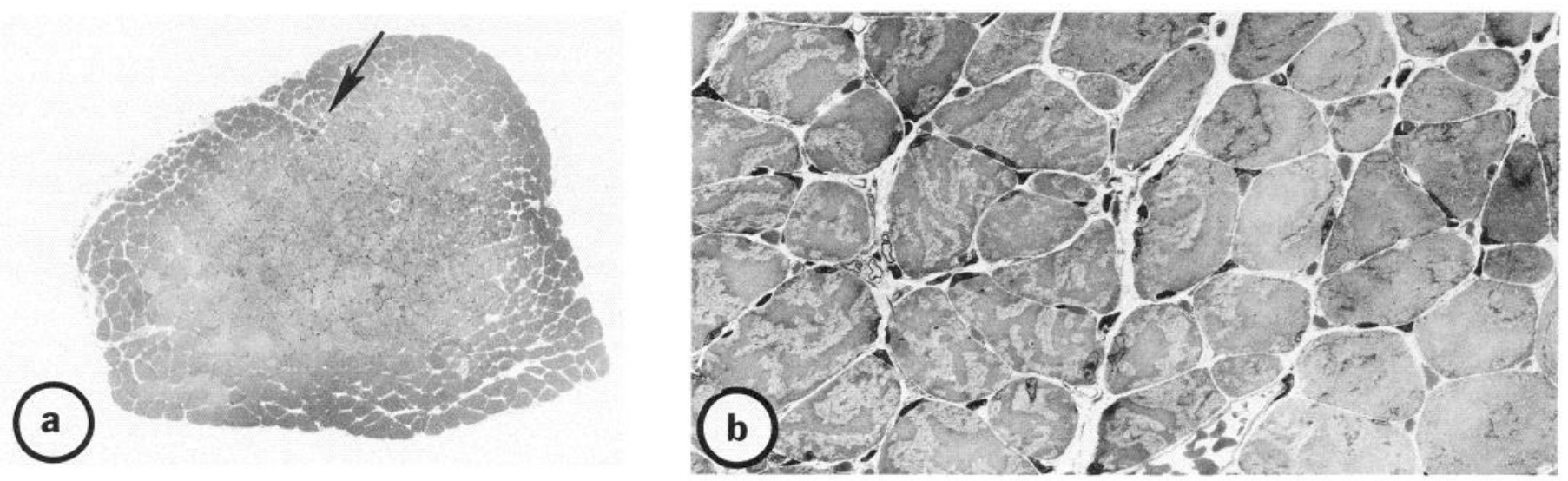

Figure 7. Denervated-reinnervated dystrophic extensor digitorum longus muscle. Denervation was performed on a 2-week-old mouse; the muscle was removed $100 \mathrm{~d}$ afterwards. The muscle is smaller in girth than the denervated-reinnervated normal muscle (compare $a$ with Fig. $6 a$ ), but is significantly larger than age-matched unoperated dystrophic muscle (Fig. $2 a$ ). Area indicated by arrow $(a)$ is enlarged in the electron micrograph $(b) . a$, Toluidine blue. $\times 60 . b$, Uranyl acetate and lead citrate. $\times 600$.

of the rat cannot be reinnervated until after the period of elimination of polyneural innervation is completed (i.e., the animal is approximately 3 weeks old; Dennis and Harris, 1980), the denervated muscle probably remains denervated for more than a week after nerve sectioning. Despite the relatively "healthy" appearance of the denervated-reinnervated dystrophic muscles, motor endplate sarcolemmas on these muscles display shallower infoldings than are found in normal denervated-reinnervated muscles. Reduction in the complexity of folding at the motor endplate region of unoperated dystrophic muscle has been previously reported (Banker et al., 1979).

The purpose of the present study was to evaluate the hypothesis that there exists for dystrophic myotubes a "window of vulnerability," requiring that they be innervated and remain innervated during a critical period of their development if they are to express the dystrophic phenotype. That nerve-muscle interaction during development might be responsible for certain features of Duchenne muscular dystrophy has also been sug-

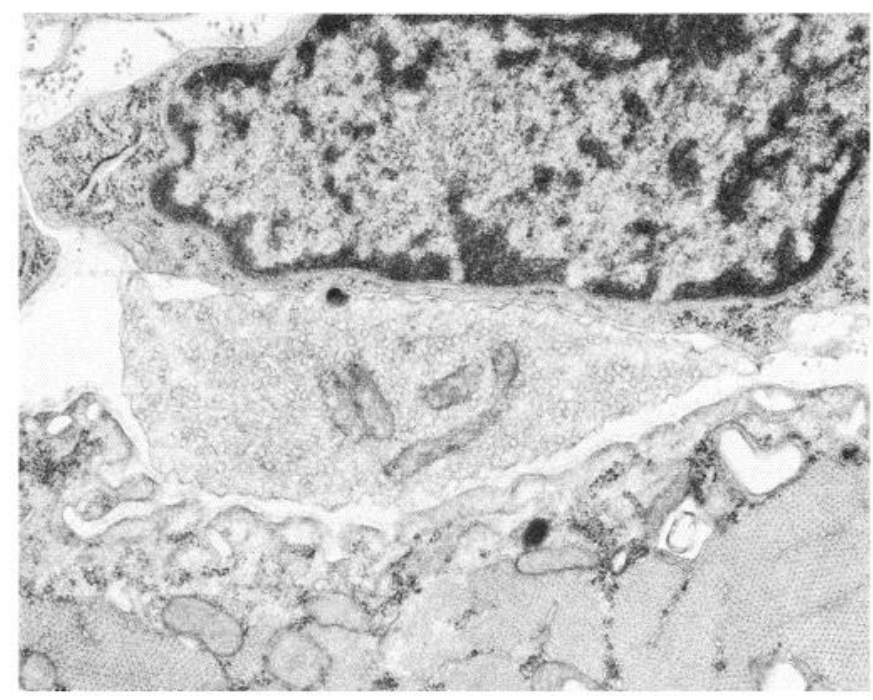

Figure 8. Motor endplate on a denervated-reinnervated dystrophic extensor digitorum longus muscle. Denervation was performed on a 2-week-old mouse, and the muscle was removed $100 \mathrm{~d}$ afterwards. The subsynaptic folds are not well developed. Compare with Figure 9. Uranyl acetate and lead citrate. $\times 17,000$. gested (Vrbova, 1983). The concept of a "critical" period for the expression of myofiber phenotype is not a new one in muscle development. It has been established that the intrafusal and extrafusal myofibers are derived from a common pool of myotubes, and that the formation of intrafusal myofibers is dependent on the presence of sensory innervation on these myotubes during a "critical" period of their development (Zelena, 1957; Zelena and Hnik, 1960). Since the phenotypic expression of intrafusal myofibers is dependent on myoneural interaction during a critical period, perhaps the phenotypic expression of muscular dystrophy is similarly dependent on myoneural interaction during a critical period following myotube formation.

The results of the present study and of our dystrophic transplantation study (Bourke and Ontell, 1986) are consistent with the "critical" period hypothesis in that, in these systems, where the innervation of myofibers relative to their time of formation is either delayed (as compared to fetal development) or interrupted shortly after myotube formation, we observe modifica-

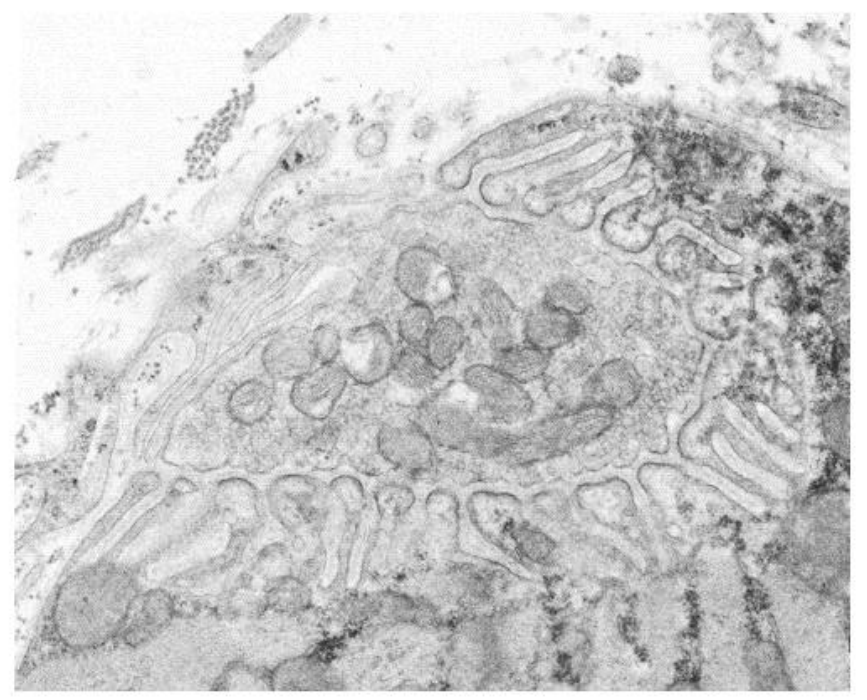

Figure 9. Motor endplate in a denervated-reinnervated normal extensor digitorum longus muscle. Denervation was performed on a 2-weekold mouse, and the muscle was removed $100 \mathrm{~d}$ afterwards. Note the depth of the subsynaptic folds. Compare with Figure 8. Uranyl acetate and lead citrate. $\times 17,000$. 
tion of the dystrophic phenotype. Further support for this hypothesis is found in a recent study $(H$. Martin and M. Ontell, unpublished observations) of regenerated dystrophic muscle formed in response to multiple injections of the myotoxic agent bupivacaine (Benoit and Belt, 1970). The bupivacaine system is similar to the transplantation system in that the myofibers undergo necrosis and are replaced by de novo formed myotubes; however, in the bupivacaine system, the myotubes become innervated immediately after they are formed (Sadeh et al., 1985), as they do during normal fetal development. In the bupivacaine system, the regenerated dystrophic myofibers express histopathological changes similar to those observed in the untraumatized dystrophic myofibers formed during fetal development. Should further experiments confirm the "window of vulnerability" hypothesis, it will be necessary to determine whether the modification of the dystrophic phenotype reflects the lack of muscle activity or the removal of some ncuronal chemical factor during a vital period of the dystrophic myotube's development. It will also be necessary to determine the precise timing of the critical period.

The "window of vulnerability" hypothesis is not the only hypothesis compatible with our observations. In our experimental systems, the phenotypic expression of murine dystrophy is modified only when the nerve to the muscle has been cut as part of the protocol. Previous studies in our laboratory have established that not all of the motor axons are successful in reinnervating grafted normal muscle (Klueber et al., 1984). It may be that not all of the motoneurons that innervate the intact dystrophic muscle are capable of reinnervating the neonatally denervated muscle. If one subscribes to the "sick" motoneuron hypothesis (McComas et a1., 1971) for an explanation of the etiology of murine dystrophy, then one may conclude that perhaps not all of the motoneurons are equally affected by muscular dystrophy. If this is true, maybe only the "healthier" motoneurons are capable of reinnervating the dystrophic muscle. There is no direct evidence, however, that there are differences within the population of motoneurons that warrant the assumption that "healthy" and "sick" motoneurons innervate any given dystrophic muscle. At the present time, we are attempting to clarify the mechanism responsible for the experimental inhibition of the dystrophic phenotype.

The failure of the dystrophic mouse to make full use of its reinnervated hindlimb is not related to the dystrophic syndrome. The normal mouse exhibits the same response to the denervation-reinnervation protocol. The inability to effectively use the limb may be related to the failure of the muscles to become reinnervated by their "appropriate" nerves. In an attempt to provide more appropriate reinnervation for the dystrophic muscles, we are currently modifying the denervation procedure by using crush lesions and by denervating closer to the muscle bellies.

\section{References}

Banker, B. Q. (1967) A phase and electron microscopic study of dystrophic muscle I. The pathological changes in the 2 week old Bar Harbor 129 dystrophic mouse. J. Neuropathol. Exp. Neurol. 26: 259275.

Banker, B. Q., and D. Denny-Brown (1959) A study of denervated muscle in normal and dystrophic mice. J. Neuropathol. Exp. Neurol. 18: $517-530$.

Banker, B. Q., N. S. Hirst, S. C. Chester, and R. Y. Fok (1979) His- tometric and electron cytochemical study of muscle in the dystrophic mouse. Ann. NY Acad. Sci. 317: 115-131.

Benoit, P. W., and W. D. Belt (1970) Destruction and regeneration of skeletal muscle after treatment with a local anesthetic, bupivacaine (Marcaine). J. Anat. 107: 547-556.

Bourke, D. L., and M. Ontell (1986) Modification of phenotypic expression of murine muscular dystrophy: A morphological study. Anat. Rec. 214: 17-24.

Bray, G. M., and A. J. Aguayo (1975) Quantitative ultrastructural studies of the axon-Schwann cell abnormality in spinal nerve roots from dystrophic mice. J. Neuropathol. Exp. Neurol. 34: 517-530.

Carlson, B. M. (1973) The regeneration of skeletal muscle-a review. Am. J. Anat. 137: 119-150.

Carlson, B. M., and E. Gutmann (1976) Free grafting of the extensor digitorum longus muscle in the rat after Marcainc pretrcatment. Exp. Neurol. 53: 82-93.

Cosmos, E., J. Butler, and A. J. Milhorat (1973) Hereditary muscular dystrophy: A possible myogenic defect in the differentiation of muscle. In Basic Research in Myology, B. A. Kakulas, ed., pp. 632-640, Excerpta Medica, Amsterdam.

Davidowitz, J., B. R. Pachter, and G. M. Breinin (1976) "Clearing" steel knife epon sections in a polystyrene film sandwich. Stain Technol. 51: $139-140$

Dennis, M. J., and A. J. Harris (1980) Transient inability of neonatal rat motoneurons to reinnervate muscle. Dev. Biol. 74: 173-183.

Gutmann, E. (1942) Factors affecting recovery of motor function after nerve lesions. J. Neurol. Neurosurg. Psychiatry 5: 81-95.

Jaros, E., and W. G. Bradley (1978) Development of the myelinated lesion in the ventral root of the dystrophic mousc. J. Ncurol. Sci. 36: 317-339.

Jaros, E., and D. Johnstone (1984) Factors affecting phenotypic expression of dystrophy in skeletal muscle of the dystrophic mouse (dy/dy). In Research into the Origin and Treatment of Muscular Dystrophy, L. P. ten Kate, P. L. Pearson, and A. M. Stadhouders, eds., pp. 103124, Excerpta Medica, Amsterdam.

Karpati, G., S. Carpenter, and S. Prescott (1982) Prevention of skeletal muscle necrosis in hamster dystrophy. Muscle Nerve 5: 369-372.

Klueber, K., J. W. Yip, and M. Ontell (1984) Size and location of the motoneuron pool supplying normal and orthotopically transplanted muscles. Brain Res. 305: 192-195.

Mauro, A. (1961) Satellite cell of skeletal muscle fibers. J. Biophys. Biochem. Cytol. 9: 493-495.

McComas, A. J., R. E. P. Sica, and M. J. Campbell (1971) "Sick" motoneurons. A unifying concept of muscle disease. Lancet 1: 321325 .

Michelson, A. M., E. S. Russell, and P. J. Harman (1955) Dystrophia muscularis: A hereditary primary myopathy in the house mouse. Proc. Natl. Acad. Sci. USA 41: 1079-1084.

Ontell, M., and K. Kozeka (1984) The organogenesis of murine striated muscle: A cytoarchitectural study. Am. J. Anat. 171: 133-148.

Ontell, M., K. C. Feng, K. Klueber, R. F. Dunn, and F. Taylor (1984) Myosatellite cells, growth, and regeneration in murine dystrophic muscle: A quantitative study. Anat. Rec. 208: 159-174.

Reynolds, E. S. (1963) The use of lead citrate at high $\mathrm{pH}$ as an electron opaque stain in electron microscopy. J. Cell Biol. 17: 208-212.

Rowe, R. W. D., and G. Goldspink (1969a) Muscle fiber growth in five different muscles in both sexes of mice. I. Normal mice. J. Anat. 104: $521-530$.

Rowe, R. W. D., and G. Goldspink (1969b) Muscle fiber growth in five different muscles in both sexes of mice. II. Dystrophic mice. J. Anat. 104: 531-538.

Sadeh, M., L. Z. Stern, and K. Czyzewski (1985) Changes in end-plate cholinesterase and axons during muscle degeneration and regeneration. J. Anat. 140: 271-293.

Sokal, L. R., and F. J. Rohlf (1981) Biometry, pp. 145-147, Freeman, San Francisco.

Vrbova, G. (1983) Duchenne dystrophy viewed as a disturbance of nerve-muscle interactions. Muscle Nerve 6: 671-675.

Zelena, J. (1957) The morphogenetic influence of innervation on the ontogenetic development of muscle spindles. J. Embryol. Exp. Morphol. 5: 282-292.

Zelena, J., and P. Hnik (1960) Irreversible elimination of muscle receptors. Nature 188: 946-947. 